Acta Crystallographica Section D

Biological

Crystallography

ISSN 0907-4449

\section{F. Xavier Gomis-Rüth* and Miquel Coll}

Institut de Biologia Molecular de Barcelona, CSIC, c/Jorda Girona 18-26, E-08034 Barcelona, Spain

Correspondence e-mail: xgrcri@ibmb.csic.es
(C) 2001 International Union of Crystallography Printed in Denmark - all rights reserved

\title{
Solving a $300 \mathrm{kDa}$ multimeric protein by low- resolution MAD phasing and averaging/phase extension
}

The structure of the conjugative coupling protein TrwB $\Delta$ N70 from Escherichia coli plasmid R388 was solved using two crystal forms. This large multimeric membrane protein of 437 residues per monomer is involved in cell-to-cell single-strand DNA transfer. Diffraction data to $2.4 \AA$ were available from trigonal crystals obtained from ammonium sulfate and to $2.5 \AA$ from monoclinic crystals grown from tartrate. A single tantalum bromide $\left(\mathrm{Ta}_{6} \mathrm{Br}_{12}^{2+}\right)$ derivative of the trigonal form, which presented a protein hexamer with $C 6$ local symmetry in the asymmetric unit, was used in a three-wavelength MAD experiment to achieve $4.5 \AA$ resolution for initial phases. Sixfold averaging and phase extension increased the effective phasing resolution and eventually produced a straightforwardly traceable electron-density map. The monoclinic structure was solved by molecular replacement, i.e. a hexamer of the trigonal form was used as a search model. Two such hexamers are present in the asymmetric unit.

\section{Introduction}

The main issue in protein crystallography is the determination of phases for the measured structure-factor amplitudes. The most widely used technique for novel structures has historically been isomorphous replacement, which consists of the introduction of atoms or ions with high scattering power into the otherwise unchanged crystal lattice, yielding detectable changes in the intensities of the diffraction patterns. This technique is susceptible to non-isomorphism between the crystal lattices of the native compound and the heavy-ion/ atom derivative prepared. However, the use of multiwavelength anomalous diffraction (MAD), which has increased with the availability of wavelength-tuneable synchrotron beam time and the emergence of crystal cryocooling techniques, has reduced this problem (González et al., 1999; Terwilliger, 1997).

Although experimental approaches have been developed to incorporate modified amino acids containing 'heavy-atom' scatterers [mainly selenomethionine (Budisa et al., 1995; Doublié, 1997)] into proteins efficiently, a large or small number of methionine residues in the asymmetric unit of crystals of large macromolecular assemblies hinders detection or renders poor phasing power, respectively. Conventional derivative preparation may also fail, since heavy ions/atoms are not easily localized within large cells owing to the excessive number of sites. In these cases, bulky ionic clusters of high scattering power can be useful and detectable using lowresolution data.

This is valid for tantalum bromide $\left(\mathrm{Ta}_{6} \mathrm{Br}_{12}^{2+}\right)$, a regular octahedron of a $4.3 \AA$ radius, successfully employed in a
Received 3 January 2001 Accepted 19 March 2001

PDB References: $\operatorname{TrwB} \Delta \mathrm{N} 70$, trigonal, 1e9r; TrwB $\Delta \mathrm{N} 70$, monoclinic, 1e9s. 
number of structural analyses (Knäblein et al., 1997). This compound introduces significant changes in the diffraction pattern of large unit cells and is formed of ions with absorption edges both with significant anomalous signals appropriate for MAD experiments and energies that can be reached at most tunable synchrotron beamlines ( $L_{\text {III }}$ edge at $1.25 \AA$ for Ta, $K$ edge at $0.95 \AA$ for $\mathrm{Br}$ ). It displays an intrinsic deep green colour that indicates putative derivatization. If the cluster does not adopt a unique conformation in the crystal lattice, the exact position of the 18 constituting ions is not determinable and so the derivative has to be treated as a single point scatterer. In these cases, the useful phasing high-resolution limit seems to be $6 \AA$ (Knäblein et al., 1997).

We applied this derivatizing agent to solve the structure of a soluble variant of $\operatorname{TrwB}$, a basic $(\mathrm{pI}=10)$ integral membrane plasmid protein encoded by the $d t r$ region of $E$. coli plasmid R388 made up of 507 residues (Llosa et al., 1994; Moncalián et al., 1999). Sequence analysis localized the transmembrane part in the first $70 \mathrm{~N}$-terminal residues (two transmembrane helices and a small periplasmic domain in between). Therefore, TrwB was overproduced and purified as a soluble fragment lacking these residues ( $\operatorname{TrwB} \Delta \mathrm{N} 70)$.

Here, we report the crystallization and the detailed structure determination protocol of 437-residue $\operatorname{TrwB} \Delta N 70$ by MAD and subsequent averaging/phase extension.

\section{Methods}

\subsection{Purification, crystallization and derivative preparation}

$\operatorname{TrwB} \Delta \mathrm{N} 70$, produced by overexpression and purified as described in Moncalián et al. (1999), was provided by G. Moncalián and F. de la Cruz. The protein was crystallized by the hanging-drop vapour-diffusion method using Linbro plates and Hampton Research Screens to obtain initial conditions.

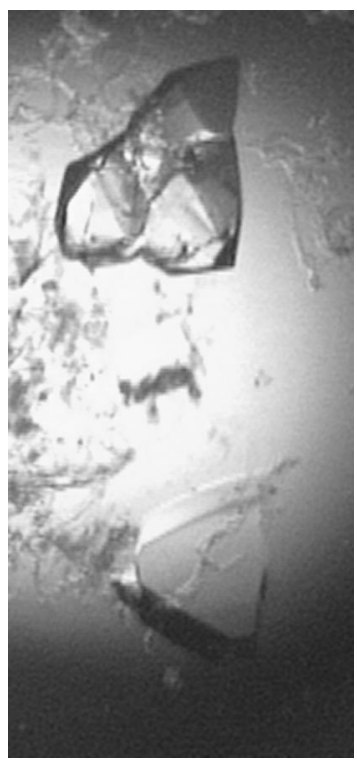

Figure 1

Crystallization drop obtained from high tartrate concentration giving rise to trigonal (upper) and monoclinic (lower) crystals simultaneously.
The best conditions consisted of equivolumetric drops $(4 \mu \mathrm{l}: 4 \mu \mathrm{l})$ of protein solution $\left(9 \mathrm{mg} \mathrm{ml}^{-1}\right)$ and $1.5 \mathrm{M}$ ammonium sulfate, $0.1 M \mathrm{NaCl}, 0.1 M$ HEPES $\mathrm{pH} 7.5$ as precipitant agent solution, which yielded well shaped trigonal crystals belonging to space group $P 3_{1} 21$ (unit-cell parameters $a=b=151.3, c=258.2 \AA$ ). In parallel, when $0.9 \mathrm{M}$ potassium/ sodium tartrate, $0.1 M$ HEPES $\mathrm{pH} 7.5$ was used as precipitant, the same trigonal crystals appeared in the drops together with a second, monoclinic $\left(P 2_{1}\right)$ crystal form (unit-cell parameters $a=107.4, b=153.4, c=162.5 \AA, \beta=94.2^{\circ}$; see Fig. 1). Some of the monoclinic crystals presented a second, smaller monoclinic cell (unit-cell parameters $a=95.2, b=155.8, c=104.7 \AA$, $\left.\beta=113.1^{\circ}\right)$. N-terminal sequencing and mass spectrometry of carefully washed and dissolved crystals revealed the expected molecular weight and sequence. Prior to data collection, cryoprotecting strategies consisted of stepwise increasing amounts of glycerol (5-20\%; crystals obtained from ammo-

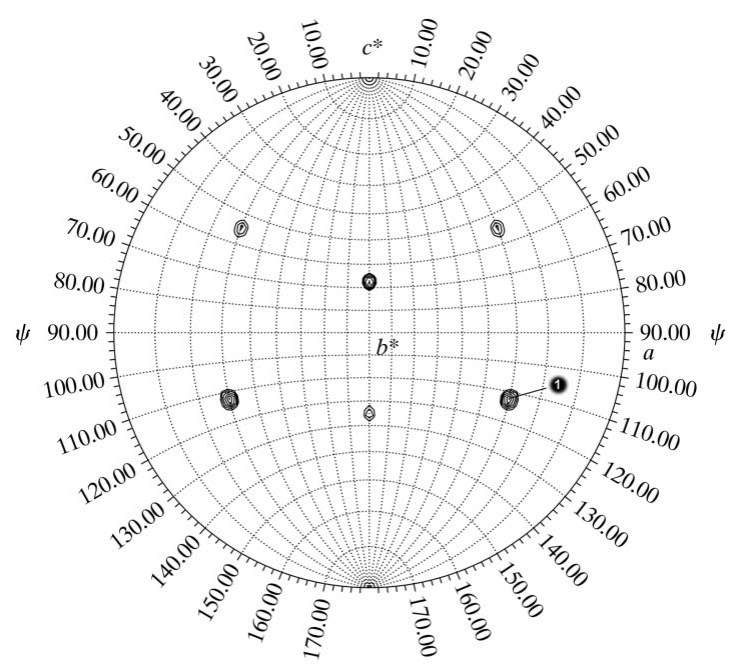

(a)

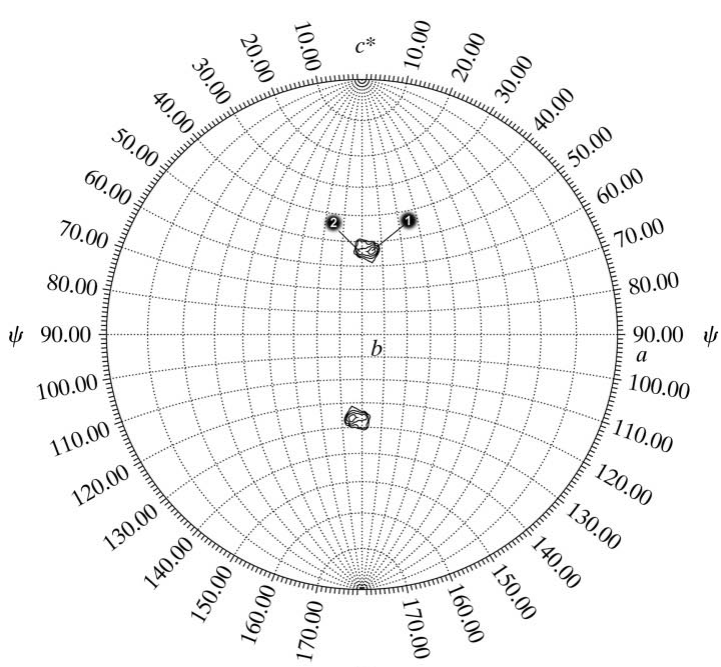

(b)

Figure 2

Self-rotation plots corresponding to section $\kappa=60^{\circ}$, as deduced from GLRF (Tong, 1993) for (a) trigonal and (b) monoclinic data. The values are $\Psi=112, \Phi=30, \kappa=60^{\circ}$ for 1 in $(a)$ and $\Psi=54, \Phi=85, \kappa=60^{\circ}$ for 1 and $\Psi=54, \Phi=91^{\circ}, \kappa=60^{\circ}$ for 2 in $(b)$. 
Table 1

Data collection and processing.

\begin{tabular}{|c|c|c|c|c|c|}
\hline Data set & $\begin{array}{l}\text { Native sulfate } \\
\text { complex }\end{array}$ & Native & $\begin{array}{l}\mathrm{Ta}_{6} \mathrm{Br}_{12}^{2+} \\
\left(f_{\max }^{\prime \prime}\right)\end{array}$ & $\begin{array}{l}\mathrm{Ta}_{6} \mathrm{Br}_{12}^{2+} \\
\left(f_{\min }^{\prime}\right)\end{array}$ & $\begin{array}{l}\mathrm{Ta}_{6} \mathrm{Br}_{12}^{2+} \\
\text { (remote) }\end{array}$ \\
\hline Space group & $P 3_{1} 21$ & $P 2_{1}$ & \multirow{2}{*}{\multicolumn{2}{|c|}{$\begin{array}{l}P 3_{1} 21 \\
a=b=151.2, c=262.8\end{array}$}} & \\
\hline Unit-cell parameters $\left(\AA{ }^{\circ}, \stackrel{ }{\circ}\right)$ & $\begin{array}{l}a=b=151.3 \\
c=258.2\end{array}$ & $\begin{array}{l}a=107.4 \\
b=153.4 \\
c=162.5 \\
\beta=94.2\end{array}$ & & & \\
\hline Wavelength $\lambda(\AA)$ & 0.844 & 1.053 & 1.254 & 1.255 & 0.827 \\
\hline No. of measurements & 1205165 & 728622 & 260537 & 317859 & 317424 \\
\hline No. of unique reflections & 133904 & 176179 & 57492 & 61615 & 67261 \\
\hline Whole resolution range $\dagger(\AA)$ & $72.6-2.40$ & $50.0-2.50$ & $48.8-3.13$ & $48.8-3.13$ & $49.4-3.00$ \\
\hline Completeness (\%) & 99.9 & 97.3 & 93.1 & 99.7 & 95.8 \\
\hline Anomalous completeness (\%) & - & - & 77.5 & 91.1 & 89.3 \\
\hline$R_{\text {merge }} \ddagger(\%)$ & 5.7 & 5.4 & 9.1 & 7.5 & 6.9 \\
\hline Average intensity $[I / \sigma(I)]$ & 9.0 & 9.9 & 3.7 & 6.8 & 5.5 \\
\hline Last resolution shell $(\AA)$ & $2.53-2.40$ & $2.64-2.50$ & $3.32-3.13$ & $3.32-3.13$ & $3.13-3.00$ \\
\hline Completeness (\%) & 99.6 & 90.0 & 60.3 & 99.1 & 72.0 \\
\hline Anomalous completeness (\%) & - & - & 28.0 & 89.9 & 44.5 \\
\hline$R_{\text {merge }} \neq(\%)$ & 28.1 & 16.9 & 33.1 & 39.7 & 30.3 \\
\hline Average intensity $[I / \sigma(I)]$ & 2.6 & 4.3 & 2.3 & 1.9 & 2.5 \\
\hline$B$ factor (Wilson) $\left(\AA^{2}\right)$ & 46.0 & 51.3 & 61.9 & 69.5 & 70.0 \\
\hline Average multiplicity & 9.0 & 4.1 & 4.5 & 5.2 & 4.7 \\
\hline$R_{\text {anomalous }} \S$ & - & - & 10.3 & 6.6 & 5.8 \\
\hline$R_{\text {deri }} \uparrow$ & - & - & 7.4 & Reference & 8.5 \\
\hline
\end{tabular}

$\dagger$ The high-resolution data collection was limited by the diameter of the detector and the large cell constants $\ddagger R_{\text {merge }}=\left[\sum_{h k l} \sum_{i}\left|I_{i}(h k l)-\langle I(h k l)\rangle\right| / \sum_{h k l} \sum_{i} I_{i}(h k l)\right] \times 100$, where $I_{i}$ is the $i$ th measurement of reflection $I(h k l)$. $\S R_{\text {anomalous }}=\sum_{h k l} \mid\langle I(h k l)\rangle-\left\langle I(\bar{h} \bar{k} \bar{l}\rangle\left|/ \sum_{h k l}[\langle I(h k l)\rangle+\langle I(\bar{h} \bar{k} l)\rangle] . \quad-R_{\text {deri }}=\sum_{h k l}\right|\left|F_{\mathrm{PH}}\right|-\left|F_{\mathrm{P}}\right|\left|/ \sum_{h k l}\right| F_{\mathrm{P}}\right|$ with respect to the data set chosen as a reference $\left(f_{\min }^{\prime}\right)$.

nium sulfate) or ethylene glycol (5-25\%; crystals produced with tartrate as precipitating agent) in mother liquor were followed. The trigonal crystals diffract to better than $2.4 \AA$ resolution and display a series of sulfate anions complexed with the protein, as revealed by the final refined structure (Gomis-Rüth et al., 2001). This structure will be referred to hereafter as the 'native sulfate complex' (see Table 1). The Matthews parameter (Matthews, 1968) suggested the presence of 4-8 monomers in the asymmetric unit (a.u.). Monoclinic crystals (termed 'native'; see Table 1) diffracted as well as the trigonal ones and were initially proposed to harbour 10-12 protomers in the a.u. A tantalum bromide $\left(\mathrm{Ta}_{6} \mathrm{Br}_{12}^{2+}\right)$ derivative was obtained by soaking native crystals overnight in a mother-liquor solution containing 5-10 $\mathrm{m} M \mathrm{Ta}_{6} \mathrm{Br}_{14}$.

\subsection{Data collection and processing}

Complete diffraction data sets were collected from a single $\mathrm{N}_{2}$-cryocooled (Oxford Cryosystems) crystal on $345 \mathrm{~mm}$ MAR Research imaging plate, $165 \mathrm{~mm}$ MAR CCD and ADSC Quantum4 CCD detectors at EMBL beamlines X31 and BW7A/B (Outstation Hamburg, DESY, Germany) and ID14-2/4 (ESRF, Grenoble, France). In particular, for a MAD experiment using the $\mathrm{Ta}_{6} \mathrm{Br}_{12}^{2+}$ derivative, the same range of $90^{\circ}\left(\Delta \Phi=1.25^{\circ}\right)$ around one rotation axis (as determined by the strategy option of MOSFLM v. 6.01; Leslie, 1991) was collected at a resolution of $3.1 \AA$ (see Table 1) at three wavelengths corresponding to the absorption maximum $(1.254 \AA)$, the inflection point $(1.255 \AA)$ and a hard remote (0.827 $\AA$ ). These values had been determined from a XANES fluorescence spectrum around the theoretical Ta $L_{\mathrm{III}}$ edge recorded from the crystal to be measured. All data were processed with MOSFLM and scaled, merged and reduced with SCALA (Evans, 1993; Table 1).

\subsection{Data analysis, structure solution and refinement}

Self-rotation calculations were performed with ncode $=1$ using data in the 10-3.5 $\AA$ range and a vector radius of $37 \AA$ with the program GLRF (Tong, 1993). The orthogonalization convention chosen was AXABZ (Tong, 1993). Initial cluster sites were determined with XPREP/ SHELXS from the SHELX 97 suite (Sheldrick, 1997; Sheldrick et al., 1993; Sheldrick \& Schneider, 1997) using the data corresponding to the threewavelength MAD experiment. A high-resolution cutoff of $6 \AA$, which allowed each cluster site to be considered as a single point scatterer, was applied. The three reduced data sets were prepared with XPREP and dispersive and anomalous sharpened difference Patterson maps were calculated. The program produced a unique reduced $\Delta F$-like data set which allowed us to determine three cluster positions by SHELXS that were consistent with the Patterson maps. These positions were refined and phases were calculated using MLPHARE (Collaborative Computational Project, Number 4, 1994). Three further positions were ascertained by means of dispersive and anomalous difference Fourier syntheses with these initial phases. Density modification was calculated with $D M$ (Cowtan \& Main, 1996) and masks with MAMA (Kleywegt \& Jones, 1999). Electrondensity syntheses were inspected and models built on a Silicon Graphics Workstation using the program TURBO-FRODO (Roussel \& Cambilleau, 1989). Model refinement and computation of $\sigma_{A}$-weighted (Read, 1986) (phased combined) maps were performed with the program suite $C N S$ v. 1.0 (Brunger et al., 1998). Molecular replacement was performed with AMoRe (Navaza, 1994).

\subsection{Miscellaneous}

Structures were superimposed with TURBO-FRODO (Roussel \& Cambilleau, 1989), LSQKAB from the CCP4 suite (Collaborative Computational Project, Number 4, 1994) and $L S Q M A N$ from the RAVE package (Uppsala Software Factory; http://alpha2.bmc.uu.se/ gerard/manuals). Electrondensity figures were prepared with TURBO-FRODO. 
Table 2

MAD phasing statistics.

\begin{tabular}{|c|c|c|c|c|c|c|}
\hline \multirow[b]{2}{*}{ Data set } & \multicolumn{2}{|c|}{$\mathrm{Ta}_{6} \mathrm{Br}_{12}^{2+}\left(f_{\max }^{\prime \prime}\right)$} & \multicolumn{2}{|c|}{$\mathrm{Ta}_{6} \mathrm{Br}_{12}^{2+}\left(f_{\min }^{\prime}\right)$} & \multicolumn{2}{|c|}{$\mathrm{Ta}_{6} \mathrm{Br}_{12}^{2+}$ (remote) } \\
\hline & Occ. & Anom. occ. & Occ. $\dagger$ & Anom. occ. & Occ. & Anom. occ. \\
\hline \multicolumn{7}{|l|}{ Heavy ion cluster sites $\ddagger \S$} \\
\hline $0.792,0.279,0.535$ & 0.29 & 4.50 & 0.0 & 2.04 & 0.45 & 1.45 \\
\hline $0.204,0.966,0.807$ & 0.24 & 2.91 & 0.0 & 1.33 & 0.41 & 1.01 \\
\hline $0.287,0.701,0.293$ & 0.10 & 1.35 & 0.0 & 0.60 & 0.16 & 0.45 \\
\hline $0.243,0.641,0.744$ & 0.09 & 1.19 & 0.0 & 0.53 & 0.19 & 0.42 \\
\hline Mean figure of merit (f.o.m.) & & & 0.63 & & & \\
\hline Phasing power (acentric) $\dagger \dagger$ & & 1.31 & & - & & 1.70 \\
\hline$R_{\text {cullis }}$ (acentric) $\ddagger$ & & 0.79 & & - & & 0.72 \\
\hline$R_{\text {cullis }}$ (anomalous) & & 0.75 & & 0.81 & & 0.87 \\
\hline
\end{tabular}

Initial NCS operators.

\begin{tabular}{|c|c|c|c|c|c|c|c|c|c|c|c|c|c|}
\hline $\mathrm{R} 1$ & 1.000 & 0.000 & 0.000 & 0.000 & 1.000 & 0.000 & 0.000 & 0.000 & 1.000 & $\mathrm{~T} 1$ & 0.000 & 0.000 & 0.000 \\
\hline $\mathrm{R} 2$ & 0.828 & 0.110 & 0.549 & -0.479 & 0.648 & 0.593 & -0.291 & -0.754 & 0.589 & $\mathrm{~T} 2$ & -109.250 & -86.281 & 126.448 \\
\hline R3 & 0.462 & -0.297 & 0.836 & -0.885 & -0.099 & 0.454 & -0.053 & -0.950 & -0.309 & $\mathrm{~T} 3$ & -135.357 & -11.008 & 307.368 \\
\hline $\mathrm{R} 4$ & 0.798 & -0.532 & -0.283 & 0.131 & 0.612 & -0.780 & 0.588 & 0.585 & 0.558 & $\mathrm{~T} 4$ & 88.409 & 168.896 & 40.839 \\
\hline R5 & 0.447 & -0.894 & -0.014 & -0.288 & -0.129 & -0.949 & 0.847 & 0.428 & -0.315 & T5 & 63.131 & 251.493 & 213.698 \\
\hline R6 & 0.255 & -0.795 & 0.550 & -0.771 & -0.510 & -0.380 & 0.583 & -0.327 & -0.744 & T6 & -46.980 & 171.308 & 342.633 \\
\hline
\end{tabular}

Refined NCS operators.

\begin{tabular}{|c|c|c|c|c|c|c|c|c|c|c|c|c|c|}
\hline R1 & 1.000 & 0.000 & 0.000 & 0.00000 & 1.000 & 0.000 & 0.000 & 0.000 & 1.000 & $\mathrm{~T} 1$ & 0.000 & 0.000 & 0.000 \\
\hline $\mathrm{R} 2$ & 0.814 & 0.118 & 0.568 & -0.507 & 0.622 & 0.598 & -0.283 & -0.775 & 0.566 & $\mathrm{~T} 2$ & -112.505 & -84.725 & 131.903 \\
\hline R3 & 0.445 & -0.271 & 0.854 & -0.895 & -0.137 & 0.423 & 0.001 & -0.953 & -0.304 & T3 & -139.019 & -1.852 & 304.090 \\
\hline $\mathrm{R} 4$ & 0.814 & -0.508 & -0.282 & 0.118 & 0.620 & -0.776 & 0.569 & 0.598 & 0.564 & $\mathrm{~T} 4$ & 86.013 & 168.227 & 40.000 \\
\hline $\mathrm{R} 5$ & 0.445 & -0.895 & 0.003 & -0.271 & -0.138 & -0.953 & 0.854 & 0.423 & -0.304 & $\mathrm{~T} 5$ & 59.561 & 251.682 & 211.616 \\
\hline R6 & 0.259 & -0.778 & 0.571 & -0.778 & -0.518 & -0.353 & 0.571 & -0.353 & -0.741 & T6 & -52.974 & 166.684 & 344.194 \\
\hline
\end{tabular}

$\dagger$ The data set at the inflection point $\left(f_{\min }^{\prime}\right)$ was taken as reference. $\$$ Fractional cell coordinates. $\S$ Occupancy and anomalous occupancy are on arbitrary scales. 9 f.o.m. $=$

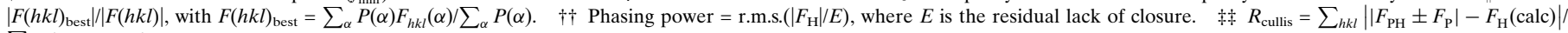
$\sum_{h k l}\left|F_{\mathrm{PH}}-F_{\mathrm{H}}\right|$

\section{Results and discussion}

\subsection{Self-rotation calculations}

The putatively high number of protomers in the asymmetric units of both crystal types suggested high-order local symmetry. Indeed, self-rotation calculations indicated a local sixfold axis at polar angles $\Psi=30, \Phi=112, \kappa=60^{\circ}$ (height $4.0 \sigma$ ) for the trigonal crystals and $\Psi=54, \Phi=85, \kappa=60^{\circ}(6.5 \sigma)$ and $\Psi=54, \Phi=91, \kappa=60^{\circ}(6.2 \sigma)$ for monoclinic data. At the same orientation, the associated twofold and threefold axes appeared as the only additional peaks. These results are consistent with the presence of six monomers displaying local C6 symmetry (hexamer) in the trigonal a.u. and two hexamers in the monoclinic a.u. (see Fig. 2). Accordingly, a putative selenomethionine derivative of TrwB would have entailed localizing 60 selenium sites (ten methionine residues per protomer, see TrEMBL access code Q04230) in the trigonal form or 120 in the monoclinic space group. Although this is not an impossible task with the methods available currently, it remains complicated.

\subsection{Initial phasing}

In order to determine the initial phases, a MAD experiment was performed with the $\mathrm{Ta}_{6} \mathrm{Br}_{12}^{2+}$ derivative of the trigonal crystal form. The intense green colour of the soaked (initially transparent and colourless) crystals contrasted with the pale colouring of the drops and was the first evidence of derivatization. Three data sets were recorded after determination of the appropriate wavelengths to optimize the anomalous signal. A total of six sites were found and refined and phases were computed for the resolution range 40-4.5 $\AA$; the overall mean figure of merit (f.o.m.; definition in Table 2) was 0.63 (see Table 2). However, these sites did not follow the local C6 symmetry found with the self-rotation function calculation (see Fig. 3a). Although the whole cluster was treated as a single point scatterer, this figure was lower but still significant in the 5.1-4.5 $\AA$ (0.49) resolution shell, in contrast to the reported limit of usefulness of $6 \AA$ (Knäblein et al., 1997).

\subsection{Density modification, averaging and phase extension}

A subsequent density-modification step (35-4.5 ^; f.o.m. = 0.79 ) assuming a solvent content of $59 \%$ (six molecules per a.u.) rendered an $F_{\text {obs }}$ electron density that was not traceable (see Fig. $3 a$ ) but allowed us to build a monomer mask because the protomer boundaries were clearly visible. A molecule of similar size to TrwB $\Delta$ N70 (procarboxypeptidase A, see Gomis-Rüth et al., 1995) was initially fitted into the density. This operation was repeated for each monomer to determine 


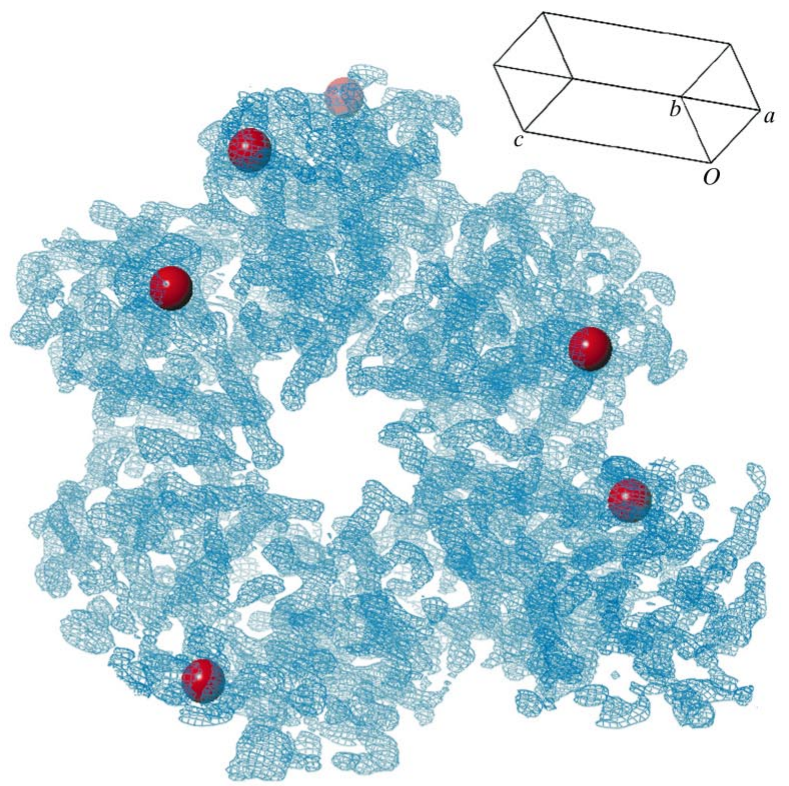

(a)
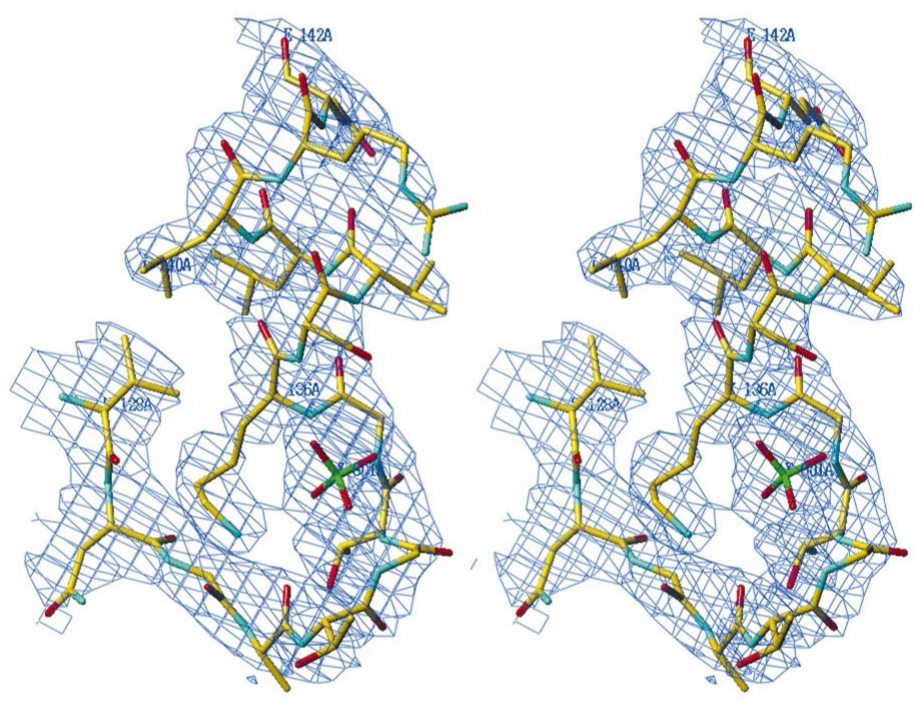

(b)
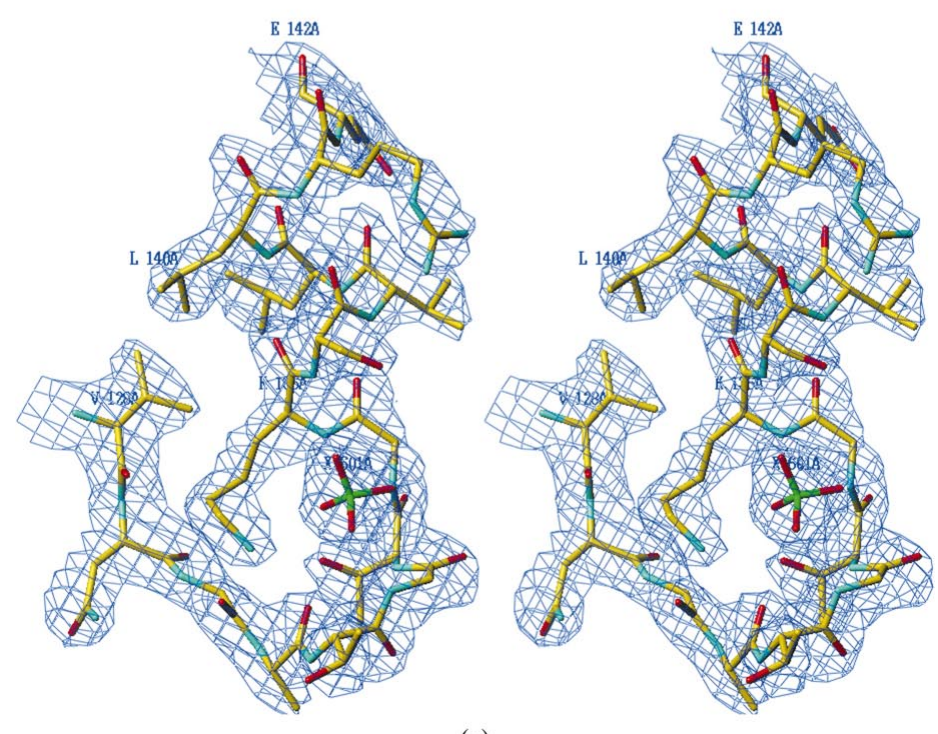

(c) preliminary parameters for the local sixfold axis and the associated approximate non-crystallographic symmetry (NCS) operators (see Table 2). These values were consistent with self-rotation calculations (see above). A second density-modification step with density averaging and phase extension under refinement of these NCS operators increased the fom to 0.75 (50-3.0 ̊). An inspection of the electron density around each cluster site revealed an almost spherical blob that hindered the precise determination of the position of the 18 constituting ions. Thus, phasing using the full resolution of the $\mathrm{Ta}_{6} \mathrm{Br}_{12}^{2+}$-derivatized crystal could not be applied. The refined NCS operators were used in the last step: the original experimental MAD phases to $4.5 \AA$ Aere directly used with the native sulfate complex structure-factor amplitudes and a last density-modification step was performed under averaging, phase extension and NCSoperator refinement starting at $5 \AA$ resolution and finally encompassing the 50-2.7 $\AA$ resolution range (final overall f.o.m. $=0.67$; see Table 2). Although the trigonal cells of the native sulfate complex and the derivative displayed a noteworthy anisomorphism ( $c=258.2$ versus $262.2 \AA$, a difference of $1.8 \%$; see Table 1 ), this effect was not significant at $5 \AA$ resolution, at which averaging and phase extension started. At this point, a straightforward traceable map was obtained (see Fig. 3b).

The electron density was interpreted and a starting model was built. Successive cycles of maximum-likelihood positional and temperature-factor refinement progressively using all data to the full resolution of $2.4 \AA$, NCS restraints, computation of phased-combined maps and manual modelling led to gradual completion of the model (see Figs. $3 b$ and $3 c$ ). The refined model has been discussed elsewhere (Gomis-Rüth et al., 2001).

\subsection{Molecular replacement to localize 40000 atoms in the monoclinic native asymmetric unit and monoclinic crystal ambiguity}

The native structure of the monoclinic crystal form was solved using data in the $15-4.5 \AA$ resolution range by molecular replacement, which confirmed $P 2_{1}$ as the correct space group. A whole TrwB $\Delta$ N70 hexamer was used as a searching model and two clear solutions were obtained at $\alpha=97.9, \beta=141.8, \gamma=293.6^{\circ}, x=0.2398$, $y=-0.0001, z=0.4666$ and $\alpha=86.3, \beta=145.2, \gamma=340.7^{\circ}$,

\section{Figure 3}

Electron-density maps corresponding to the trigonal crystal form. (a) Initial $F_{\text {obs }}$ density contoured at $2 \sigma$ calculated with experimental phases using data to $4.5 \AA$ resolution after density modification but prior to averaging, viewed along the local sixfold axis. Red spheres denote the position of the six heavy-ion cluster sites. The unit cell displayed in the upper right corner orientates the C6 macromolecule cluster. $(b)$ Interpretable $F_{\text {obs }}$ density contoured at $1.2 \sigma$ computed using experimental phases after density modification, averaging and phase extension to $2.7 \mathrm{~A}$. (c) Final $\sigma_{A}$-weighted (Read, 1986) $2 F_{\text {obs }}-F_{\text {calc }}$ type map (1.2 $\sigma$ contour) to $2.4 \AA$ resolution. The latter two maps further display the final refined model superimposed on the density as sticks. 


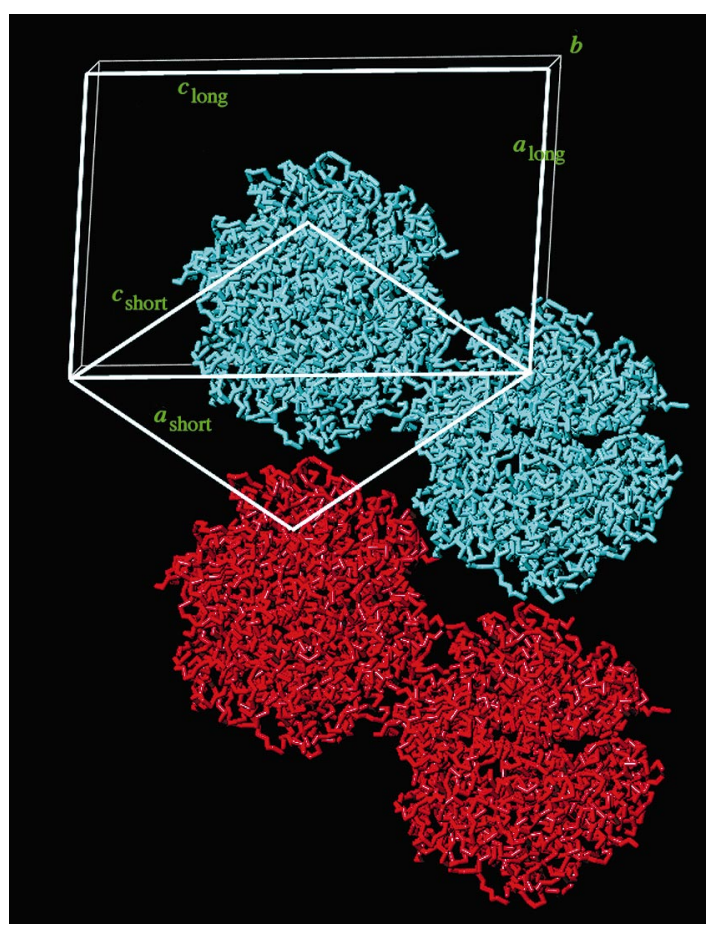

Figure 4

Monoclinic ambiguity. Monoclinic crystals randomly presented two cells (see \$3.4). The transformation of the two molecules present in the largecell asymmetric unit corresponds to a rotation of $8^{\circ}$ and a translation of $93 \AA$. In the small unit cell, this rotation is reduced to a pure cell translation and both molecules belong to different unit cells.

$x=0.7450, y=0.9884, z=0.9687$ (where $\alpha, \beta$ and $\gamma$ are Eulerian angles and $x, y$ and $z$ are in fractional cell coordinates) after 'fiting' $\left[\mathrm{CC} / R_{\text {factor }}\right.$ (for definition, see Navaza, 1994) equal to $67.4 \% / 36.3 \%$; second highest solution $41.2 \% /$ $47.1 \%$; calculated after positioning and rigid-body refinement of both hexamers]. This agrees with the presence of 12 monomers arranged as two hexamers per a.u. The model was inspected and the new electron-density-based differences with the trigonal structure were corrected. The structure has been described elsewhere (Gomis-Rüth et al., 2001).

The two hexamers in the monoclinic a.u. are related by a quasi-pure (local) translation of $93 \AA$ and a slight relative rotation of $8^{\circ}$, in agreement with the two close peaks found with the self-rotation calculation (see $\$ 3.1$ ), just separated by $6^{\circ}$ in $\Phi$. This finding explains why some monoclinic crystals presented a smaller cell with unit-cell parameters $a=95.2$, $b=155.8 \AA, c=104.7 \AA, \beta=113.1^{\circ}$ and therefore only one hexamer per a.u. This small unit cell is related to the large one in such a way that the $a$ and $c$ axes of the latter are the diagonals of the former, with equivalent $b$ axes (see Fig. 4). The two hexamers found in the large monoclinic cell would be interconnected by one cell translation along the $a$ axis in the short-cell crystals.

We thank Gabriel Moncalián and Fernando de la Cruz for providing TrwB $\Delta$ N70, Robert Huber for supplying tantalum bromide and Isabel Usón for help with XPREP/SHELXS. We are also indebted to Rosa Pérez-Luque for assistance in crystallization experiments and to Ana González for synchrotron data collection. This study was supported by grants BIO2000-1659, PB98-1631 and 2FD97-0518 from the Ministerio de Educación y Cultura, Spain, and by grant 1999SGR188 and the Centre de Referència en Biotecnologia, both from the Generalitat de Catalunya. Support was also available from the ESRF and the European Commission Improving Human Potential Programme, Contract No. HPRI1999-00017, and from EU TMR/LSF grant ERBFMGCECT980134 to the EMBL Hamburg Outstation and ERBFMGECT980133 to the EMBL Grenoble Outstation. The ESRF, Grenoble, France is acknowledged for the provision of synchrotron-radiation facilities.

\section{References}

Brunger, A. T., Adams, P. D., Clore, G. M., DeLano, W. L., Gros, P., Grosse-Kunstleve, R. W., Jiang, J.-S., Kuszewski, J., Nilges, M., Pannu, N. S., Read, R. J., Rice, L. M., Simonson, T. \& Warren, G. L. (1998). Acta Cryst. D54, 905-921.

Budisa, N., Steipe, B., Demange, P., Eckerskorn, C., Kellermann, J. \& Huber, R. (1995). Eur. J. Biochem. 230, 788-796.

Collaborative Computational Project, Number 4 (1994). Acta Cryst. D50, 760-763.

Cowtan, K. D. \& Main, P. (1996). Acta Cryst. D52, 43-48.

Doublié, S. (1997). Methods Enzymol. 276, 523-530.

Evans, P. (1993). Proceedings of the CCP4 Study Weekend. Data Collection and Processing, edited by L. Sawyer, N. Isaacs \& S. Bailey, pp. 114-122. Warrington: Daresbury Laboratory.

Gomis-Rüth, F. X., Gómez, M., Bode, W., Huber, R. \& Avilés, F. X. (1995). EMBO J. 14, 4387-4394.

Gomis-Rüth, F. X., Moncalián, G., Pérez-Luque, R., González, A., Cabezón, E., de la Cruz, F. \& Coll, M. (2001). Nature (London), 409, 637-641.

González, A., Pédelacq, J.-D., Solà, M., Gomis-Rüth, F. X., Coll, M., Samama, J.-P. \& Benini, S. (1999). Acta Cryst. D55, 1449-1458.

Kleywegt, G. J. \& Jones, T. A. (1999). Acta Cryst. D55, 941-944.

Knäblein, J., Neuefeind, T., Schneider, F., Bergner, A., Messerschmidt, A., Löwe, J., Steipe, B. \& Huber, R. (1997). J. Mol. Biol. 270, $1-7$.

Leslie, A. G. W. (1991). Crystallographic Computing V, edited by D. Moras, A. D. Podjarny \& J. C. Thierry, pp. 27-38. Oxford University Press.

Llosa, M., Bolland, S. \& de la Cruz, F. (1994). J. Mol. Biol. 235, 448464.

Matthews, B. W. (1968). J. Mol. Biol. 33, 491-497.

Moncalián, G., Cabezón, E., Alkorta, I., Valle, M., Moro, F., Valpuesta, J. M., Goñi, F. M. \& de la Cruz, F. (1999). J. Biol. Chem. 274, 36117-36124.

Navaza, J. (1994). Acta Cryst. A50, 157-163.

Read, R. J. (1986). Acta Cryst. A42, 140-149.

Roussel, A. \& Cambilleau, C. (1989). Silicon Graphics Geometry Partners Directory, edited by Silicon Graphics, pp. 77-79. Mountain View, CA, USA: Silicon Graphics.

Sheldrick, G. M. (1997). Methods Enzymol. 276, 628-641.

Sheldrick, G. M., Dauter, Z., Wilson, K. S., Hope, H. \& Sieker, L. C. (1993). Acta Cryst. D49, 18-23.

Sheldrick, G. M. \& Schneider, T. R. (1997). Methods Enzymol. 277, 319-343.

Terwilliger, T. C. (1997). Methods Enzymol. 276, 530-537.

Tong, L. (1993). J. Appl. Cryst. 26, 748-751. 